\title{
INFLUNCE OF SOME ADJUVANTS ON EFFICACY OF CERTAIN INSECTICIDES AGAINST ONION THRIPS, Thrips tabaci ( LIND.) INFESTING ONION AND GARLIC PLANTS Abd-Allah, A. A. A. \\ Plant Protection Res. Inst., Agric. Res. Center, Dokki, Giza, Egypt
}

\begin{abstract}
The effect of two natural agents (as adjuvants) on the efficiency of some insecticides (biofly, malathon, nudrin and challenger) were determined by using pneumatic knapsack motor sprayer(Kubota)at $95 \mathrm{~L} . /$ fed. against nymphal and adult stages of Thrips tabaci infesting onion and garlic plants. The additives used were bio new film(bnf) and glue. The results indicated an increase of efficiency of the tested insecticides as a result of mixing with these adjuvants. The effect of(malathon, nudrin, challenger and biofly after malathon) at 0.75 recommended rate on the initial percent reduction nymphs and adult stages of T.tabaci infesting onion at the $1^{\text {st }}$ season seemed to exceed the recommended rate when mixed with (bnf) which caused $59.79,59.05,61.84,51.13$ and $59.3,78.0,67.5,48.71 \%$, respectively .

The same trend were occurred approximately at the $2^{\text {nd }}$ season. Also, similar results were recorded on garlic plants at the two studied seasons with slightly increase of efficacy. Bio new film exhibited an increase of initial percent reduction, but glue increased the residual effect. In the respect to the yield of onion and garlic, the highest yield were recorded for plots treated with biofly(3/4R)after malathon(3/4R) mixed with glue $\left(12.69,12.15\right.$ and12.34,12.64 ton $/ \mathrm{fed}$. ) at $1^{\text {st }}$ and $2^{\text {nd }}$ seasons, respectively, while the lowest yield was recorded for plots were treated with biofly which recorded 8.87,8.56 and 7.87,7.98 ton/fed., respectively. Therefore, it could be decided that the adjuvants (glue and bnf) mixed with the tested insecticides(3/4R)lead to high efficacy.
\end{abstract}

\section{INTRODUCTION}

Onion Allium cepa L. and Garlic Allium sativum L. are considered among the most important field crops cultivated in the world .Both crops liable to attack with many insect pests from seedlings until harvest causing severe damage to the plants and yield. Thrips tabaci lind. is the most damaging insect pest of onion and garlic in all Egyptian regions (Sabra et al., 2007). Because of consuming the fresh green onion and also to avoid chemical control problems, many attempts have been carried out to use nonchemical origin insecticides as a potential components of integrated pest management (Goodwin et al., 2002).

Chemical pesticides are still primary means of pest control ,but their use is becoming more controversial. The concept of biocides is being ecofriendlly as well as environmental safe and specificity to most of the target pests (Girgis,2003). Natural products from various plants are a rich source of bioactive substances, which have been exploited as insecticides and or / biologically active components against insect pests (Abo-Sholoa,1990).Spray adjuvants are chemicals that usually form a part of the formulated insecticides, although, in some circumstances they are added to insecticides tank mix prior to application. These additives may improve mixing with diluting ,improve insecticide activity in the field (Omar et al., 2003). Addition 
Abd-Allah, A. A. A.

of some adjuvants to pesticide spray solution cause a change in physicochemical properties and enhance of pesticide efficiency (Nagwa and ELSise, 1999).

Consequently, use of certain adjutants with synthetic pesticides may decrease their rates of application (EL-Metwally et al.,1991). Richard (1974) stated that thickening agents increased the viscosity of spray solution, therefore they reduced the drift and increased the sticking and insecticidal efficiency. Furmidg (1962) concluded that wetting agents decreased the surface tension of spray solution then increased the wet ability ,spreading and depositing on treated surfaces.

The aim of the present work is to study the effect of some local available additives on insecticidal efficiency of biofly, malathon (57\%), nudrin (90\%SP), challenger(36\%SC) against onion thrips attacking onion and garlic plants.

\section{MATERIALS AND METHODS}

Two additive agents, new bio (bnf) film and glue(g), were added to four known insecticides, biofly, malathon, nudrin, challenger to control T. tabaci on onion (Giza 20) and garlic(Balady) plants. The insecticides were used when the population of thrips was high, at the first half of March 2008 and 2009 seasons .An area of $1 / 2$ feddan for both crop receiving normal agriculture practices was divided into 45 plots of $42 \mathrm{~m} 2$ each. Two rows between each plots was included as guard rows to prevent the drift of spray solution. Six samples of 10 plants each ( before spraying and at 2,5,8,11 and 14 days after spraying) for each replicate were randomly chosen ,and firstly examined in the field then put in paper bags and examined again in the laboratory using a stereomicroscope to count the existing individuals of thrips inhabiting the apical of plants. Initial effect (2 days after spray ) and residual effect(means of $5,8,11$ and 14 days after spray) was calculated according to Henderson and Tilton equation(1955).General effect was the means of $2,5,8,11$ and 14days after spray. Treatments were arranged in a randomized complete block design. Pneumatic knapsack motor sprayer(Kubota)at95L./ Fed. was used.

\section{A. The test insecticides :}

1.Biocide:

Entomofungus Beauveria bassiana (Ballsamo):Biofly $\quad\left(2.3 \times 10^{7}\right.$ conidia/ml) $400 \mathrm{ml} / \mathrm{fed}$.

\section{2-Chemical insecticides :}

* Malathon (malathion 57\% E.C.), diethyl (dimethoxy thiophosphor ylthio) succinate. It was provided by Adwia Co. ( ADWIA) $10^{\text {th }}$ of Ramadan. Used at $1.0 \mathrm{~L} . /$ fed.

* Nudrin (Methomyl 90\% S.P.) N-CC(methylamino) carbonyl oxy ethanimidothioate It was provided by BASFCO. Germay. Used at 300 $\mathrm{gm} . / \mathrm{fed}$.

3-The insect growth regulator compound (Challenger 36\% S.C.). Common name : Chlorfenapyr . 
Chemical name: 4-bromo-2-(4- $\alpha$-lorophenyl)-1-ethoxymethyl-5- trifluoro methylpyrole-3- carbonilrile. It was provided by BASFCO. Germay. $100 \mathrm{ml}$ / fed.

B. Additives used:

1.Bio new film(bnf) (spreading and nutrient material) containing $2 \% \quad \mathrm{p}_{2} \mathrm{O}_{5}+$ $3 \% \mathrm{k}_{2}+9 \%$ (spreading and wetting agent)produced by Misr El-Dawliea for Agriculture Devlopment .

2.Glue (granules) supplied by El-Sabaa Co., Cairo and used at $3 \%$.

Tank mix method was used for mixing the two tested adjuvants (bnf and g) with the insecticides at the rates of $2 \%$ and $3 \%$,respectively. One type of water was used,the calculated dose of the insecticide was added. Control plots were sprayed with water only .

\section{RESULTS AND DISCUSSION}

The initial and residual effects of the tested insecticides (biofly, malathon ,nudrin and challenger) either alone at the recommended rate or in combination with the tow tested adjuvants bio new film (bnf) and glue (g) against nymphs and adults of Thrips tabaci infesting onion and garlic plants could be discussed as follows:

\section{Onion plants (first season) :}

\section{The initial effect:}

Results in Table (1) indicated that there were significant differences not only between the tested compounds when tested singly but also between them when mixed with the adjuvants. The highest initial effect against nymphs was occurred for the mixture of (challenger +bnf) which recorded $61.84 \%$ reduction. However ,the role of the tested adjuvants on the efficiency of toxicants was compounds against the adults was higher in most cases than that against nymphs whether the compounds tested singly or in combination with the two additives .The effect of adjuvants against adults was more obvious than that against nymphs. General effect of the tested insecticides against nymphs and adults was relatively close from each other without any specific effect for these toxicants against the tested insect. Also, the role of the tested adjuvants was relatively limited but they saved $1 / 4$ of the recommended rates Abd-Allah(1999)found that the Beauveria bassiana gave poor control against whitefly nymphs infesting cucumber plant.

\section{The residual effect:}

The residual effect was calculated as a mean of total effect after $5,8,11$ and 14 days from spraying .

It is clear from the results of Table (1) that the glue adjuvant slightly increased the residual mortality when added to all tested compound of biofly, malathon, nudrin, challenger and (biofly + malathon) which caused $72.72 \%$, $72.22 \%, 82.27 \%, 75.91 \%$ and $86.72 \%$ reduction against nymphal stage and gives $70.05 \%, 76.12 \%, 74.45 \%, 74.9 \%$ and $88.99 \%$ reduction against adult stage, respectively, similar trend were also recorded for the other adjuvant against both nymphes and adults (Table 1). Sticker in the spray solution at the rate of $500 \mathrm{ml} /$ feddan protected carbaryl residual from rapid decrease 
.(EL-Sayed et al.,1969. As for the general effect, the highest percent reduction was occurred for biofly $(3 / 4 R)+$ malathon $+b n f(b+m+b n f)$ against nymphs and biofly $(3 / 4 R)+$ malathon +glue $(b+m+g)$ against adults which recorded 79.41 and $79.1 \%$ reduction of population, respectively. Similar results were reported by Betana et al. (2004) who found that the inclusion of some adjuvant (glow) in spray emulsions of esfenvalerate and profenfos at their full and half recommended field rates, enhanced the activity of the tested insecticides at half recommended rate.

Table (1): Efficiency of the tested insecticides at the recommended rate or at the $3 / 4$ rcommended rate plus the two adjuvants against nymphs and adults of T.tabaci infesting onion plants and yield at 2008 season .

\begin{tabular}{|c|c|c|c|c|c|c|c|c|}
\hline \multirow[b]{2}{*}{ Treatments } & \multirow[b]{2}{*}{$\begin{array}{l}\text { Rate of } \\
\text { application/ } \\
\text { fed }\end{array}$} & \multicolumn{3}{|c|}{ Nymphs } & \multicolumn{3}{|c|}{ Adults } & \multirow[b]{2}{*}{ Yield } \\
\hline & & $\begin{array}{c}\text { Initial } \\
\text { Effect } \\
\%\end{array}$ & $\begin{array}{l}\text { M. of } \\
\text { resid. \% }\end{array}$ & $\begin{array}{c}\text { General } \\
\text { effect } \\
\%\end{array}$ & \begin{tabular}{|c|} 
Initial \\
effect \\
$\%$
\end{tabular} & $\begin{array}{l}\text { M. of } \\
\text { resid. } \\
\%\end{array}$ & $\begin{array}{c}\text { Geneal. } \\
\text { effect } \\
\%\end{array}$ & \\
\hline Biofly(R) & $400 \mathrm{ml} / \mathrm{fed}$ & $24.48^{n}$ & $67.6 \mathrm{e}$ & $58.98 \mathrm{~h}$ & $43.75 \mathrm{~g}$ & $63.38 d$ & $59.31 \mathrm{e}$ & $8.87 \mathrm{f}$ \\
\hline Biofly $(3 / 4 R)+$ bnf & $300 \mathrm{ml} / \mathrm{fed}$ & $31.75^{9}$ & $70.65 \mathrm{de}$ & $62.83 \mathrm{~g}$ & $44.29 \mathrm{~g}$ & $68.38 \mathrm{c}$ & $63.56 \mathrm{~d}$ & 9.10 \\
\hline Biofly(3/4R)+glue & $300 \mathrm{ml} / \mathrm{fed}$ & $38.32 f$ & $72.72 \mathrm{~cd}$ & $65.84 \mathrm{fg}$ & $48.95 f$ & $70.05 c$ & $65.83 d$ & 9.15 ef \\
\hline Malathon(R) & 1L./fed. & $54.47^{\circ}$ & 70.32de & $67.75 \mathrm{ef}$ & $41.07 \mathrm{~h}$ & $70.12 \mathrm{c}$ & 64.31d & $9.48 \mathrm{e}$ \\
\hline Malathon(3/4R)+ bnf & & $59.79^{\text {ab }}$ & $72.58 \mathrm{CD}$ & $70.02 \mathrm{cde}$ & $59.3 d$ & $75.5 \mathrm{~b}$ & $72.26 \mathrm{bc}$ & $10.45 d$ \\
\hline Malathon $(3 / 4 \mathrm{R})+$ glue & & $54^{\mathrm{C}}$ & $72.22 \mathrm{CD}$ & 68.58def & $58.76 \mathrm{~d}$ & $76.12 b$ & $72.7 \mathrm{bc}$ & $11.20 \mathrm{c}$ \\
\hline Nudrin(R) & $300 \mathrm{ml} / \mathrm{fed}$ & $56.28^{c}$ & $73.78 \mathrm{CD}$ & $70.28 \mathrm{cde}$ & $64.47 \mathrm{c}$ & $73.59 \mathrm{bc}$ & $71.77 \mathrm{bc}$ & $11.35 \mathrm{c}$ \\
\hline Nudrin(3/4R)+ bnf & & $59.05 b$ & $81.82 b$ & 77.27ab & $78 \mathrm{a}$ & $69.72 \mathrm{c}$ & $71.38 \mathrm{bc}$ & $11.98 \mathrm{~b}$ \\
\hline Nudrin $(3 / 4 \mathrm{R})+$ glue & & $56.12 \mathrm{c}$ & $82.27 b$ & 77.04ab & $69.85 b$ & $74.45 b$ & $73.53 b$ & $12.0 \mathrm{~b}$ \\
\hline Challenger $(\mathrm{R})$ & $100 \mathrm{ml} / \mathrm{fed}$ & $54.41 \mathrm{c}$ & 70.86de & 67.91ef & $53.85 \mathrm{e}$ & $72.96 \mathrm{bc}$ & $69.14 \mathrm{C}$ & $10.35 \mathrm{~d}$ \\
\hline Challenger(3/4R)+ bnf & & $61.84 a$ & $74.01 \mathrm{~cd}$ & 71.57cde & $67.5 \mathrm{~b}$ & $73.95 \mathrm{bc}$ & $72.66 \mathrm{bc}$ & $11.78 \mathrm{~b}$ \\
\hline Challenger $(3 / 4 \mathrm{R})+$ glue & & $56.17 \mathrm{c}$ & $75.91 \mathrm{c}$ & $71.96 \mathrm{cde}$ & $64.07 \mathrm{c}$ & $74.9 \mathrm{~b}$ & $72.73 b c$ & $11.9 \mathrm{~b}$ \\
\hline $\begin{array}{l}\text { Biofly(3/4R)+ } \\
\text { Malathon(3/4R) }\end{array}$ & & $45.98 \mathrm{e}$ & $80.59 b$ & $73.66 \mathrm{~cd}$ & $47.86 f$ & $85.86 a$ & $78.26 a$ & $12.3 \mathrm{a}$ \\
\hline \begin{tabular}{|l} 
Biofly(3/4) \\
Malathon $(3 / 4 \mathrm{R})+$ bnf
\end{tabular} & & $51.13 d$ & $86.48 a$ & $79.41 a$ & $48.71 \mathrm{f}$ & $76.41 b$ & $70.87 \mathrm{bc}$ & $12.11 \mathrm{~b}$ \\
\hline $\begin{array}{l}\text { Biofly(3/4R)+ } \\
\text { Malathon }(3 / 4 \mathrm{R})+\text { glue }\end{array}$ & & $49.64 d$ & $86.72 a$ & $79.3 a$ & $39.58 \mathrm{~h}$ & $88.99 a$ & $79.1 \mathrm{a}$ & $12.69 \mathrm{a}$ \\
\hline Check treatment & & & & & & & & $8.21 \mathrm{~g}$ \\
\hline & & & & & & 3.786 & 3.222 & 0.359 \\
\hline
\end{tabular}

\section{Onion plants (second season ) :}

\section{The initial effect:}

Data in Table (2) revealed that the highest initial effect was occurred by (challenger +bnf) against nymphal and adult stages which recorded 80.08 and $75.81 \%$ reduction, respectively.

\section{The residual effect :}

The results in Table (2) indicated that the addition of glue to the tested compounds (except challenger) were more active than other mixtures with respect to their efficacy against thrips nymphs which recorded the highest reduction percentages, $70.66 \%, 66.09 \%, 76.68 \%, 76.08 \%$ and $78.22 \%$ reduction, for biofly, malathon, nudrin and mixture of malathon +biofly respectively. 
The same results were also occurred against adult stage of thrips except biofly when mixed with glue.The present results go in line with those reported by Nagwa and EL-Sisi 1999) who founds that the effect of Merlene and Sumialpha at half recommended rate plus on the initial mortality of the $2^{\text {nd }}$ instar larvae of Spodoptera littoralis seemed to be nearly to that of the complete recommended rate. It seems also that biofly induced a high residual effect. The obtained data agree with those obtained by EL-Mezayyen et al. (2003) who stated that fenvalerate when mixed with biofly and kz oil, and atabron alone exhibited high reduction against jassid which caused $92.19,87.92$, and $83.71 \%$ reduction, respectively.

Table (2): Efficiency of the tested insecticides at the recommended rate or at the $3 / 4$ rcommended rate plus the two adjuvants against nymphs and adults of $T$. tabaci infesting onion plants and yield at 2009 season .

\begin{tabular}{|c|c|c|c|c|c|c|c|c|}
\hline \multirow[b]{2}{*}{ Treatments } & \multirow[b]{2}{*}{$\begin{array}{l}\text { Rate of } \\
\text { Application } \\
\text { /fed. }\end{array}$} & \multicolumn{3}{|c|}{ Nymphs } & \multicolumn{3}{|c|}{ Adults } & \multirow[b]{2}{*}{ Yield } \\
\hline & & $\begin{array}{c}\text { Initial } \\
\text { Effect } \\
\%\end{array}$ & $\begin{array}{l}\text { M. of } \\
\text { resid. } \\
\%\end{array}$ & \begin{tabular}{|c|} 
General \\
effect \\
$\%$
\end{tabular} & $\begin{array}{c}\text { Initial } \\
\text { Effect } \\
\%\end{array}$ & $\begin{array}{l}\text { M.of } \\
\text { resid. } \\
\%\end{array}$ & $\begin{array}{l}\text { General } \\
\text { effect } \%\end{array}$ & \\
\hline Biofly(R) & $400 \mathrm{ml} / \mathrm{fed}$ & $32.71^{\mathrm{h}}$ & $58.35 \mathrm{~h}$ & $52.72 \mathrm{~h}$ & $28.5 \mathrm{k}$ & 71.93efg & $63.24 \mathrm{e}$ & $8.56 \mathrm{~g}$ \\
\hline Biofly $(3 / 4 \mathrm{R})+$ bnf & $300 \mathrm{ml} / \mathrm{fed}$ & $33.00^{h}$ & $61.1 \mathrm{gh}$ & $55.48 \mathrm{~g}$ & $29.83 \mathrm{k}$ & 73.87de & 65.06 & $8.91 \mathrm{fg}$ \\
\hline Biofly $(3 / 4 \mathrm{R})+$ glue & $300 \mathrm{ml} / \mathrm{fed}$ & $41.83^{\mathrm{tg}}$ & $70.68 \mathrm{e}$ & $64.91 \mathrm{e}$ & $33.15 \mathrm{j}$ & 72.82ef & $64.88 \mathrm{de}$ & $10.15 \mathrm{e}$ \\
\hline Malathon(R) & 1L./fed. & $41.24 \mathrm{~g}$ & $60.8 \mathrm{gh}$ & $56.89 \mathrm{~g}$ & $51.25 \mathrm{~h}$ & $69.97 \mathrm{fg}$ & $65.41 \mathrm{de}$ & $9.01 \mathrm{f}$ \\
\hline $\begin{array}{l}\text { Malathon }(3 / 4 R)+ \\
\text { bnf }\end{array}$ & & $44.75 f$ & $63.37 f g$ & $59.81 f$ & $64.45 \mathrm{ef}$ & $74.16 \mathrm{de}$ & $72.22 \mathrm{c}$ & $9.15 \mathrm{f}$ \\
\hline Malathon $(3 / 4 \mathrm{R})+$ glue & & $43.53^{\mathrm{fg}}$ & $66.09 f$ & $61.58 f$ & $59.58 \mathrm{~g}$ & 76.66cde & $74.45 c$ & $10.10 \mathrm{e}$ \\
\hline Nudrin(R) & $300 \mathrm{gm} / \mathrm{fed}$. & $68.13 d$ & 71.81de & $71.01 \mathrm{~cd}$ & $67.5 \mathrm{~cd}$ & $77.27 \mathrm{bcd}$ & $75.32 b c$ & $11.63 \mathrm{bc}$ \\
\hline Nudrin(3/4R)+bnf & & $78.26 a b$ & $75.46 a b c$ & $71.02 a$ & $74.74 a$ & $78.94 \mathrm{bc}$ & $78.1 \mathrm{ab}$ & $12.03 \mathrm{ab}$ \\
\hline Nudrin(3/4R)+glue & & $70.7 d$ & 76.68ab & 75.48ab & $70.11 \mathrm{~b}$ & $79.06 \mathrm{bc}$ & $77.27 a b$ & 11.95ab \\
\hline Challenger(R) & $100 \mathrm{ml} /$ fed. & $76.29 b$ & 71.87de & $72.75 c$ & $62.63 f$ & $68.47 \mathrm{~g}$ & $67.3 d$ & $10.82 \mathrm{~d}$ \\
\hline Challenger(3/4R)+ bnf & & $80.08 a$ & $76.08 a b$ & $76.87 a$ & $75.81 \mathrm{a}$ & 72.5ef & $73.17 \mathrm{c}$ & $11.71 \mathrm{bc}$ \\
\hline Challenger $(3 / 4 \mathrm{R})+$ glue & & $73.45 c$ & $72.84 \mathrm{cde}$ & $72.96 \mathrm{bc}$ & 65.92de & 73.11ef & $71.67 \mathrm{c}$ & $11.43 \mathrm{C}$ \\
\hline \begin{tabular}{|l} 
Biofly $(3 / 4 \mathrm{R})+$ \\
Malathon $(3 / 4 \mathrm{R})$
\end{tabular} & & $44.41 f$ & 75.44abc & $69.23 d$ & $45.4 i$ & $79.52 b$ & $72.7 c$ & $12.04 \mathrm{ab}$ \\
\hline \begin{tabular}{|l|} 
Biofly $(3 / 4)+$ \\
Malathon $(3 / 4 \mathrm{R})+b n f$
\end{tabular} & & $55.62 \mathrm{e}$ & $74.3 \mathrm{bcd}$ & $70.57 \mathrm{~cd}$ & $68.33 \mathrm{bc}$ & $83.18 a$ & $80.21 \mathrm{a}$ & $11.63 \mathrm{co}$ \\
\hline \begin{tabular}{|l|} 
Biofly $\quad(3 / 4 \mathrm{R})$ \\
Malathon $(3 / 4 \mathrm{R})+$ glue
\end{tabular}$+$ & & $53.36 \mathrm{e}$ & $78.22 \mathrm{a}$ & $73.25 \mathrm{bc}$ & $63.44 f$ & $84.82 \mathrm{a}$ & $80.54 a$ & $12.15 \mathrm{a}$ \\
\hline Check treatment & & & & & & & & $8.05 \mathrm{~h}$ \\
\hline L.S.D.0.05\% & & 2.734 & 2.888 & 2.436 & 2.237 & 3.39 & 3.444 & 0.370 \\
\hline
\end{tabular}

\section{Garlic plants (first season):}

\section{The initial effet:}

Data in Table (3) revealed that, the two tested adjuvants increased the efficiency of the tested compounds against both stages of $T$. tabaci in compared with the compounds alone. The highest mixture was nudrin + bnf while biofly $(R)$ was the lowest one against nymphs and adult stages. Similar results were obtained by Ahmed (1992) who reported that the initial and residual activities of malathon either alone or in binary mixtures with zylex foliar fertilizer induced high initial and bio-residual activity against thrips infesting cucumber, vegetable marrow and tomato. It shows that, binary mixtures of biofly and malathon + adjuvants were not effective (antagonist) against the insects. 
Abd-Allah, A. A. A.

Table (3): Efficiency of the tested insecticides at the recommended rate or at the $3 / 4$ rcommended rate plus the two adjuvants against nymphs and adults of T.tabaci infesting garlic plants and yield at 2008 season.

\begin{tabular}{|c|c|c|c|c|c|c|c|c|}
\hline \multirow[b]{2}{*}{ Treatments } & \multirow[b]{2}{*}{$\begin{array}{c}\text { Rate of } \\
\text { application }\end{array}$} & \multicolumn{3}{|c|}{ Nymphs } & \multicolumn{3}{|c|}{ Adults } & \multirow[b]{2}{*}{ Yield } \\
\hline & & $\begin{array}{c}\text { Initial } \\
\text { effect } \\
\%\end{array}$ & $\begin{array}{c}\text { M.of } \\
\text { resid.\% }\end{array}$ & $\begin{array}{l}\text { General } \\
\text { effect } \%\end{array}$ & $\begin{array}{c}\text { Initial } \\
\text { effect } \\
\%\end{array}$ & $\begin{array}{c}\text { M.of } \\
\text { resid. \% }\end{array}$ & $\begin{array}{l}\text { General } \\
\text { effect } \%\end{array}$ & \\
\hline Biofly(R) & $\mathrm{ml} / \mathrm{fed} .400$ & $13.48 \mathrm{j}$ & $62.09 c$ & $47.46 \mathrm{~g}$ & $12.84 \mathrm{k}$ & $52.29 \mathrm{~g}$ & $44.39 \mathrm{~g}$ & $7.87 f$ \\
\hline Biofly $(3 / 4 \mathrm{R})+$ bnf & $\mathrm{ml} /$ fed. 300 & $19.02 \mathrm{i}$ & $55.96 \mathrm{de}$ & $55.3 \mathrm{de}$ & $24.45 i$ & $63.19 f$ & $55.44 f$ & $9.0 \mathrm{e}$ \\
\hline Biofly(3/4R)+glue & $300 \mathrm{ml} / \mathrm{fed}$. & $14.31 \mathrm{j}$ & $56.75 d$ & $48.26 \mathrm{~g}$ & $19.17 \mathrm{j}$ & $53.38 \mathrm{~g}$ & $46.54 \mathrm{~g}$ & $8.8 \mathrm{e}$ \\
\hline Malathon(R) & 1L./fed. & $55.62 c$ & 53.73ef & $48.19 \mathrm{~g}$ & $38.57 \mathrm{~h}$ & $66.18 \mathrm{ef}$ & $60.66 \mathrm{e}$ & $9.05 \mathrm{e}$ \\
\hline Malathon(3/4R)+ bnf & & $59.62 b$ & $49.47 \mathrm{~g}$ & 54.89de & $43.91 \mathrm{f}$ & $71.96 \mathrm{~cd}$ & $66.75 \mathrm{~cd}$ & $10.2 \mathrm{~d}$ \\
\hline Malathon(3/4R)+glue & & $51.76 \mathrm{de}$ & $58.19 d$ & $56.9 \mathrm{~cd}$ & $43.07 \mathrm{~g}$ & $72.9 c$ & $66.93 \mathrm{~cd}$ & $11.3 \mathrm{~b}$ \\
\hline Nudrin(R) & $300 \mathrm{gm} / \mathrm{fed}$. & $52.73 d$ & $47.4 \mathrm{~g}$ & $48.47 \mathrm{fg}$ & $52.61 \mathrm{~cd}$ & $68.47 \mathrm{de}$ & $65.99 \mathrm{~d}$ & $10.3 \mathrm{~d}$ \\
\hline Nudrin(3/4R)+ bnf & & $65.73 a$ & $47.26 \mathrm{~g}$ & $50.95 \mathrm{fg}$ & $54.11 c$ & $74.47 \mathrm{bc}$ & $70.4 \mathrm{bc}$ & $10.98 \mathrm{bc}$ \\
\hline Nudrin(3/4R)+glue & & $56.55 c$ & $52.16 f$ & 53.06ef & 48.07ef & $77.47 a b$ & 71.59ab & $12.17 \mathrm{a}$ \\
\hline Challenger(R) & $100 r$ & $47.29 f g$ & $64.8 \mathrm{bc}$ & $61.3 a b$ & $61.54 \mathrm{~b}$ & $72.14 \mathrm{~cd}$ & $70.02 \mathrm{bcd}$ & $12.01 \mathrm{a}$ \\
\hline Challenger $(3 / 4 \mathrm{R})+$ bnf & & $56.61 \mathrm{c}$ & $62.98 \mathrm{bc}$ & $61.71 a b$ & $66.97 a$ & $77.17 a b$ & $75.13 a$ & $11.98 \mathrm{a}$ \\
\hline Challenger(3/4R)+glue & & $52.85 d$ & $65.46 \mathrm{~b}$ & $62.89 a$ & $54.52 c$ & $78.57 a$ & 73.72ab & $12.19 \mathrm{a}$ \\
\hline $\begin{array}{|ll|}\text { Biofly } \quad(3 / 4 \mathrm{R}) & + \\
\text { Malathon }(3 / 4 \mathrm{R})\end{array}$ & & $46.56 \mathrm{~g}$ & $62.12 \mathrm{c}$ & $59.01 \mathrm{bc}$ & $46.34 f$ & $62.35 f$ & $59.15 e$ & $10.8 \mathrm{c}$ \\
\hline \begin{tabular}{|l|} 
Biofly $(3 / 4)+$ \\
Malathon $(3 / 4 \mathrm{R})+$ bnf
\end{tabular} & & $49.83 e f$ & $65.23 \mathrm{bc}$ & $62.15 a b c$ & $50.46 \mathrm{de}$ & $70.49 \mathrm{~cd}$ & $66.49 \mathrm{~cd}$ & $12.0 \mathrm{a}$ \\
\hline $\begin{array}{l}\text { Biofly }(3 / 4 \mathrm{R}) \\
\text { Malathon }(3 / 4 \mathrm{R})+\text { glue }\end{array}+$ & & $43.29 \mathrm{~h}$ & 68.89a & $63.77 a$ & $46.17 f$ & $65.03 e f$ & $61.26 \mathrm{e}$ & 12. \\
\hline Check treatment & & & & & & & & $7.23 \mathrm{~g}$ \\
\hline L.S.D. $0.05 \%$ & & 2.623 & 2.534 & 3.363 & 2.398 & 3.721 & 3.708 & 0.36 \\
\hline
\end{tabular}

Generally the binary mixture of myco-insecticide plus malathon at its recommended rate gave acceptable to good control till the experimental end time after 12 days.

\section{The residual effect:}

Data presented in Table (3) showed generally the tested compounds and their mixtures with the tested additives were more toxic to adults than to nymphs. challenger and nudrin I recorded the highest residual effect against adults but that biofly $(R)$ against both nymphs and thrips adults was occurred the lowest mean residual effect specially against adults but challenger only against nymphs. The efficiency was enhancement(synergist) when biofly was mixed with malathon and both glue or bnf which recorded $68.89 \%$ and 65.23 in nymphs \& $65.03 \%$ and $70.49 \%$ reduction in adults, respectively. Abd-Allah,(1999) conducted in field experiments to evaluate the efficacy of smallest amounts from carbosulfan (I.e.0.75 and $0.5 \mathrm{R}$ ) in the mixture latent the good performance of the mixture to exert good control of whitefly nymphs and adult) on cucumber plants. Also, Ford and Salt (1990), reported that the manner in which an agro-chemical is deposited on plant surface can determine its effectiveness as a crop protectant.

Data in Table (3) revealed generally challenger was the most effective compound against nymphs and adult of thrips attacking garlic whether to used alone or combined with the two additives. Abd EL-All (1988) illustrated that, beside the basic active ingredient, adjuvant (Polyethylene glycol-600mono oleate, Egyptool B.I.M. $25 \%$ and Calcium dodecyl benzene 
sulfonate)are used to increase the biological activity of pesticides. They are included in almost all commercial pesticide formulations.

\section{Garlic plants (second season) :}

Results in Table (4) indicated generally that bnf and glue when combined with all the tested insecticides induced high level of reduction of insect population as initial effect or as residual effect.

Table (4): Efficiency of the tested insecticides at the recommended rate or at the $3 / 4$ rcommended rate plus the two adjuvants against nymphs and adults of T.tabaci infesting garlic plants and yield at 2009 season.

\begin{tabular}{|c|c|c|c|c|c|c|c|c|}
\hline \multirow{2}{*}{ Treatments } & \multirow{2}{*}{$\begin{array}{l}\text { Rate of } \\
\text { application }\end{array}$} & \multicolumn{3}{|c|}{ Nymphs } & \multicolumn{3}{|c|}{ Adults } & \multirow[b]{2}{*}{ Yield } \\
\hline & & $\begin{array}{l}\text { Initial } \\
\text { effect } \%\end{array}$ & $\begin{array}{l}\text { M.of } \\
\text { resid.\% }\end{array}$ & $\begin{array}{l}\text { General } \\
\text { effect\% }\end{array}$ & \begin{tabular}{|c|} 
Initial \\
effect $\%$
\end{tabular} & \begin{tabular}{|c|} 
M.of \\
resid. \%
\end{tabular} & $\begin{array}{l}\text { General } \\
\text { effect\% }\end{array}$ & \\
\hline$\overline{B i o f l y}(\mathrm{R})$ & $\mathrm{ml} / \mathrm{fed} .400$ & $31.4^{81}$ & $76.93^{\mathrm{d}}$ & $67.84^{\dagger}$ & $36.72^{1 !}$ & $71.91^{\dagger}$ & $64.92^{9}$ & 7.98 \\
\hline Biofly $(3 / 4 R)+$ bnf & $\mathrm{ml} /$ fed. 300 & $40.17^{n}$ & $80.9^{c}$ & $72.75^{\mathrm{e}}$ & $40^{\prime}$ & $79.43^{\text {cde }}$ & $71.54^{\mathrm{e}}$ & $9.34 \mathrm{~h}$ \\
\hline Biofly $(3 / 4 \mathrm{R})+$ glue & $300 \mathrm{ml} / \mathrm{fed}$ & $41.26^{h}$ & $75.27^{\mathrm{d}}$ & $68.43^{\dagger}$ & $34 .^{15]}$ & $75.52^{\mathrm{et}}$ & $67.36 f^{9}$ & $9.0 \mathrm{i}$ \\
\hline Malathon(R) & 1L./fed. & $59.92^{\dagger}$ & $77.08^{\mathrm{d}}$ & $73.65^{\mathrm{e}}$ & $55.81^{9}$ & $71.46 f$ & $68.33 \mathrm{e}^{\mathrm{fg}}$ & $9.21 \mathrm{hi}$ \\
\hline Malathon $(3 / 4 \mathrm{R})+\mathrm{bnf}$ & & $73.34^{b}$ & $87.32^{b}$ & $84.52^{\mathrm{bc}}$ & $70.68^{c}$ & $72.39 f$ & $72.04^{\mathrm{e}}$ & $10.68 \mathrm{fg}$ \\
\hline Malathon(3/4R)+glue & & $67.13^{\mathrm{e}}$ & $87.5^{\mathrm{b}}$ & $83.43^{\mathrm{C}}$ & $65.86^{\mathrm{d}}$ & $73.02 f$ & $71.59^{\mathrm{e}}$ & $10.62 \mathrm{~g}$ \\
\hline Nudrin(R) & $\mathrm{gm} / \mathrm{fed} .300$ & $58.27^{c}$ & $90.97^{\mathrm{a}}$ & $84.43^{\mathrm{bc}}$ & $58.11^{9}$ & $80.85^{\text {bcd }}$ & $67.3^{\mathrm{d}}$ & $10.7 \mathrm{fg}$ \\
\hline Nudrin(3/4R)+ bnf & & $72.16^{\mathrm{bc}}$ & $93.75^{\mathrm{a}}$ & $89.34^{\mathrm{a}}$ & $64.34^{\mathrm{ed}}$ & $83.19^{b c}$ & $79.42^{\text {cd }}$ & 10.91ef \\
\hline Nudrin(3/4R)+glue & & $67.32 \mathrm{e}$ & $94.55^{\mathrm{a}}$ & $89.1^{a}$ & $59.0^{\text {tg }}$ & $89.65^{a}$ & $83.52^{b}$ & $12 a b c$ \\
\hline Challenger(R) & $\mathrm{ml} /$ fed. 100 & $68.05^{\mathrm{de}}$ & $78.3^{\text {cd }}$ & $77.79^{\mathrm{d}}$ & $74.42^{D}$ & $84.95^{\mathrm{D}}$ & $82.84^{\mathrm{DC}}$ & $11.73 \mathrm{~d}$ \\
\hline Challenger(3/4R)+ bnf & & $70.3^{\mathrm{d}}$ & $80.62^{c}$ & $78.56^{\mathrm{d}}$ & $79.87^{a}$ & $90.55 a$ & $88.41^{\mathrm{a}}$ & $11.83 \mathrm{bcd}$ \\
\hline Challenger(3/4R)+glue & & $68.82^{\mathrm{de}}$ & $81.68^{c}$ & $79.1^{d}$ & $72.31^{\mathrm{bc}}$ & $92.8^{\mathrm{a}}$ & $88.7^{\mathrm{a}}$ & $12.06 a b$ \\
\hline $\begin{array}{|lr|}\text { Biofly } \quad(3 / 4 \mathrm{R}) \\
\text { Malathon(3/4R) } \\
\end{array}$ & & 54.9 & $91.64^{\mathrm{a}}$ & $86.97^{\mathrm{a}}$ & $45.52^{h}$ & def & $.95 \mathrm{ef}$ & $11.0 \mathrm{e}$ \\
\hline $\begin{array}{ll}\text { Biofly } & (3 / 4) \\
\text { Malathon }(3 / 4 \mathrm{R})+\text { bnf }\end{array}$ & & 7 & $91.62^{\mathrm{a}}$ & $88.93^{\mathrm{ab}}$ & $62.05^{\mathrm{ef}}$ & $.91^{\text {cde }}$ & $76.33^{d}$ & $11.8 \mathrm{~cd}$ \\
\hline \begin{tabular}{|l} 
Biofly $\quad(3 / 4 \mathrm{R})$ \\
Malathon $(3 / 4 \mathrm{R})+$ glue
\end{tabular} & & $72.58^{\mathrm{bc}}$ & $91.7^{a}$ & $88.54^{\mathrm{a}}$ & $69.82^{c}$ & $82.94^{\mathrm{bc}}$ & $80.31^{\text {bcd }}$ & 12.6 \\
\hline Check treatment & & & & & & & & $7.62 \mathrm{k}$ \\
\hline L.S.D. $0.05 \%$ & & 2.436 & 2.363 & 3.044 & 3.335 & 4.213 & 3.729 & 0.225 \\
\hline
\end{tabular}

Generally effect of the tested insecticides when mixed with the adjuvants exceeded pronouncedly that induced when used singly whether against nymphs or against adults . It is also showed that reduction percentages recorded in this season were relatively higher than that of the previous season (2008). These results agree with those obtained by Bargar (1984) were found that sprays of methoxychlor at various concentrations with applied by a hydrolic sprayer to American elemis, Ulmus Americana in different seasons and localities were significantly affected by addition of stickers. The obtained results agree with those of Brady et al.,(1980) who reported that sticker adjuvant including oxal,new film 17,plant card ,plyace stretcher and triton $x-100$ could be used for increasing the persistence of chlorpyrifos and chlorpyrifos-methyl. 
Abd-Allah, A. A. A.

\section{Effect of treatments on yield :}

\section{On onion:}

Data in Tables (1 and 2) showed that the yield of all treated plots were increased significantly in comparable with untreated ones. It could be classified into three categories, the $1^{\text {st }}$ for mixture of (biofly and malathon) and nudrin plus both bnf, glue with yield about 11.63 and 12.69 ton/fed. for the two seasons, respectively; the $2^{\text {nd }}$ which occupied malathon + bnf and biofly +glue with about 9.01 and 10.45 ton/fed. for the two seasons, respectively; the last group had the rest treatments (between 8.56 - 9.0 ton/fed.).

\section{On garlic :}

The relatively high yield of garlic was occurred for plots treated with malathon mixed with both bnf or glue, nudrin, challenger and (biofly + malathon) ranged between 10.3- 12.34 ton/fed. compared with $7.23-7.62$ ton/fed. for untreated plots during the two seasons while the rest compounds ranged between 7.87-10.2 ton/fed. at two seasons.

Finally the adjuvants have the ability to increase viscosity of spray liquids and significantly reduce small droplets. Therefore, they are successful thickening agents for flowable formulations (Abd El-All,1988). The high potency of the glue mixed with some tested compounds may be due to adhesive force of the droplets of the aqueous solution on the treated surfaces accordingly the deposit ratio increase Frear,1955).Also,(Soliman,2002) found that, viscosity ratio increased when carbosulfan at half recommended rate was mixed with glue. On the other hand, Bio new film (spreading, wetting and nutrient materials) enhanced the system defense in the plant. (AbdAllah,1999)found that the binary mixture of the biofly plus foliar fertilizer (Stumo-Green) which containing $10 \%$ potassium and $1.5 \%$ phosphorus induced high residual activity against whitefly infesting cucumber. The effect of both Merlene and Sumialpha at half recommended rate of application plus glue against Spodoptera littoralis seemed to be nearly to that of the complete recommended rate with free additives, this changes in insecticides properties led to increase their retention and insecticides efficiency (Nagwa et al. 1999). It could be also, concluded that controlling $T$. tabaci depends mainly on the adjuvants with little rates of insecticides. These information's indicated that, the \%reductions were resulted from the arrival and homogenous coverage of spraying solution to the places inhabits the immature stages of the thrips.

\section{REFERENCES}

Abd-Allah, A.A.A. (1999).Effect of certain chemical and bio-control agents on whitefly, Bemicia tabaci (Geen.) infesting cucumber. Ph.D. Thesis, Fac. of Agric., Zagazig Univ. ,Egypt.

Abd El-All, S.M. (1988). The role of some additives in improving effectiveness and performance of pesticide formulations. M.S.Thesis ,Fac. Of Agric., Cairo Univ.

Abo-Sholoa, M.K.A.E. (1990). Naturally occurring chemicals for pest control in comparing with some synthetic pesticides, Ph.D. Thesis, Fac. of Agric., Kafr El- Seikh, Tanta Univ. 
Ahmed, S.A. (1992). Studies on some pests infesting certain vegetable crops. M.Sc. Thesis, Fac. of Agric. ,Zagazig Univ., Egypt.

Barger, J.H. (1984). Evaluation of hydrolically applied methoxychlor to protect American elemes from feeding by the smaller Europen elem bark beetle (Coloptera : Scolytidae ). J. Econ. Entomol., 77:794-797.

Betana, M.D.;M.A. Hussein and A.M.A. EL-Kadi (2004). Influence of some adjuvant on physico-chemical properties, effectiveness, and pesticides formulations. J. Agric. Sci., Mansoura Univ., 29(4): 2105 - 2116.

Brady, V.E.; C.W. Berisford; T.L. Hall and J.S. Hamiton (1980). Efficacy and persistence of chloropirophos, chloropirophos-methyl and lindane for preventive and remedial control of the southern pine beetle. J. Econ. Entomol., 73:639-641.

EL-Metwally, H.E.; H.S.M. Fahmy ;Z.M. EL-Attal and S.M. Abdel-All (1989). The role of some local additives in improving effectiveness and

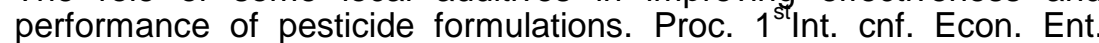
(11\}:189-201.

EL-Mezayyen G.A.; A.M.A. Nassef and I.I. EL-Fakharany (2003). Effect of certain alternative compoundsw and their combinations with fenvalerate on some cotton insect pests and their associated predators, J. Agric. Sci., Mansoura Univ.28(4):3061-3069 .

EL-Sayed, M.M., Shahin, A. and R.H. Bishara (1969). The effect of foliar sticker on increasing the residual activity of Sevin. Bull. Ent. Soc. Egypt, Econ. Ser., 3:47-53.

Frear, D.E.H. (1955). Chemistry of the pesticide. In: D. Van Nostrand Co. Inc., Toronto, New York, London, Third Ed., 460pp .

Furmidge, C.G.L. (1962). Physico-chemical studies on agricultural sprays: The retention of spray liquids on leaf surface. J. Sc. Food. Agric., 13:27-140 .

Henderson, C.E. and E.T. Telton (1955): Tests with acaricides against the brown wheat mite. J. Econ. Ent.,48:157-161.

Girgis, N.R. (2003). Studies on various control methods of the potato tuber moth Phothrima operculella (Zeller). Thesis Ph.D. Fac. of Agric. Cairo Univ., Egypt.

Goodwin, S.; Steiner, M. and E. Enkegard (2002). Developments in IPM for protected cropping in Australia. Bulletin -OILB.SROP. 25:7,81-84 .

Nagwa A.B. and A.G.EL-Sisi (1999). Using certain additives tp improve the physical properties and residual activity of some pesticides against the cotton leaf warm Spodoptera littoralis (Boisd.), Egypt J. Appl. Sci.; 14(3): 310-322

Omar, A.E.; M.M. Amir; A.A. EL-Fishawy and A.E. El-Sobky (2003). Laboratory and field evaluation of malathion and certain adjuvant against the faba bean aphid, Aphis craccivora (Koch), Zagazig J. Agric. Res.,30 (5): 2021-2032.

Richardson, R.C.(1974).Control of spray drift with thickening agents. J. Agric. Engn. Res. $19: 227-231$.

Sabra, I.M.A.; M. A. EL-Nagar and M.S. Shalaby (2007).Further ecological studies on onion pests in Egypt, Egypt .J.Agric.,85(4):1259-1266.

Soliman, M.H.A. (2004).Studies on main insect pests infesting cowpea plants in Sharkia Governorate. Ph D. Thesis, Fac. of Agric. Zagazig Univ. 
Abd-Allah, A. A. A.

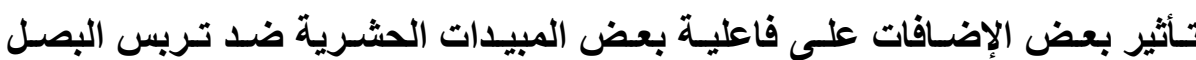

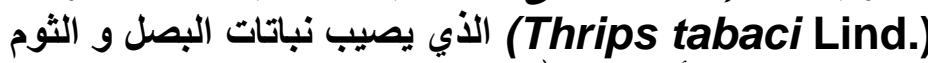

عاطف عبد الفتاح أحمد عبدالله

معهر بحوث وقاية النباتات ـ مركز البحوث الهُ البحوث الزراعية ــ دقي - جيزة ـ مصر

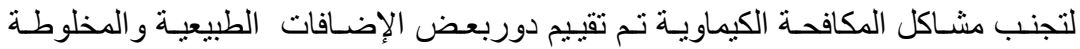

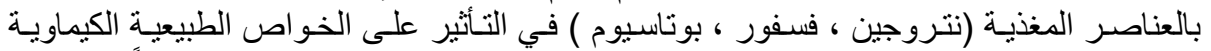

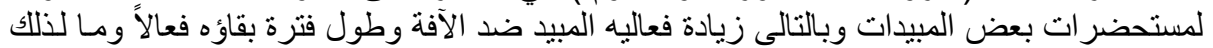
من آثار إقتصادية وبيئية جيدة 0

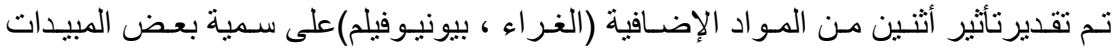

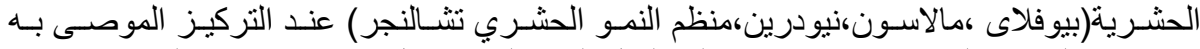

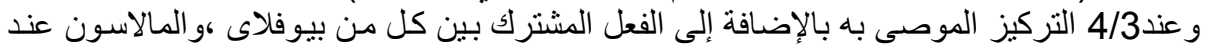

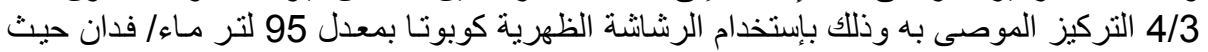

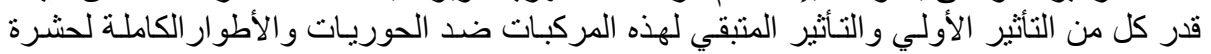

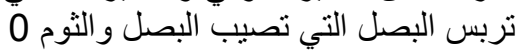

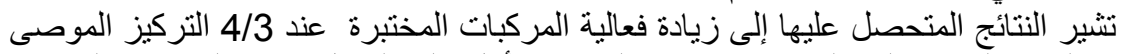

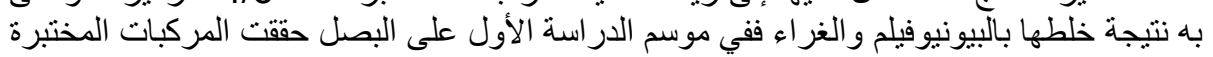

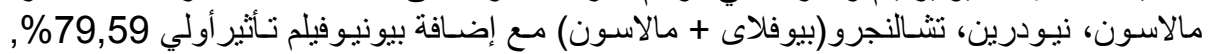

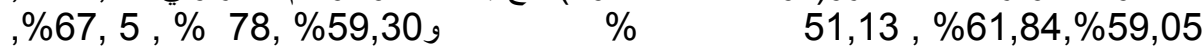

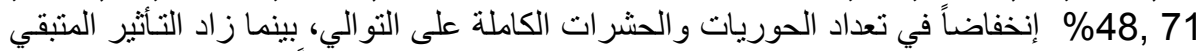

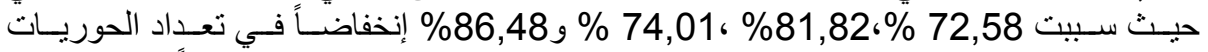
و الحشر ات الكاملة على التو الي وفي الموسم الثاني كانت النتائج في نفس المسار تقنريباً.

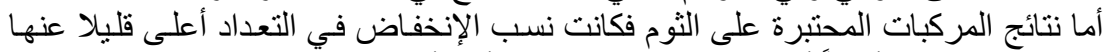

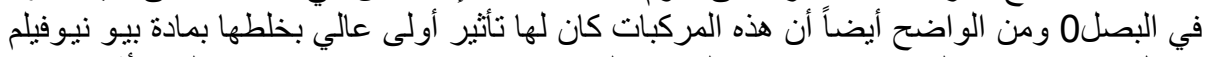

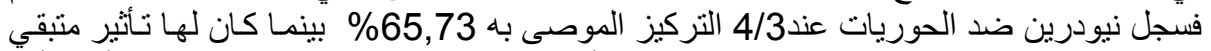

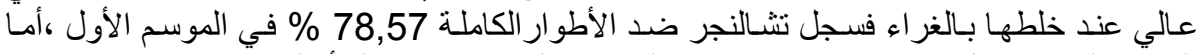

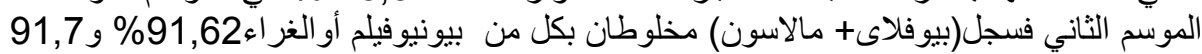

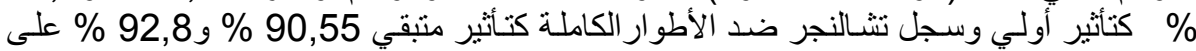

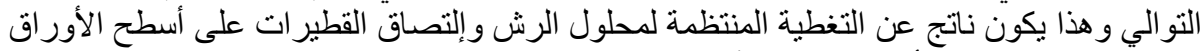
المعاملة والوصول إلى أماكن تواجد الآند الآفة 0

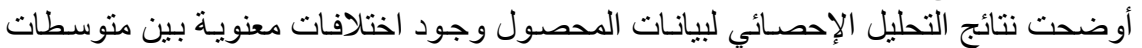

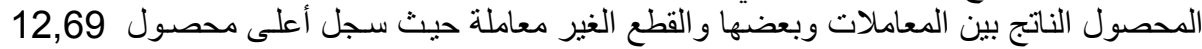

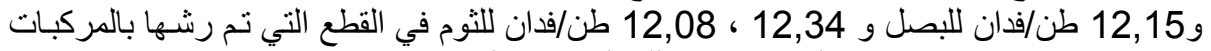

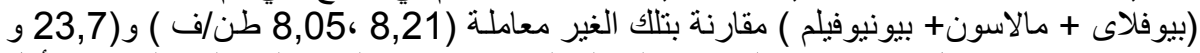

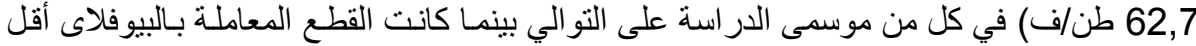

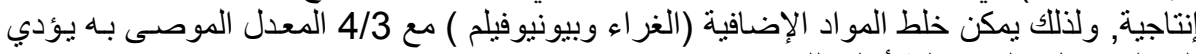
إلى الحصول على فاعلية أعلى للمركب.

كلية الزراعة - جامعة المنصورة كلية الزراعة - جامعة الزقازيق جامعة الزفوة

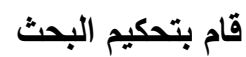

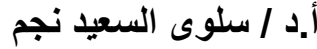

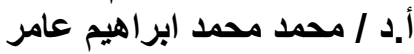

